\title{
Ultrahigh frequency oscillations and multimode dynamics in vertical cavity surface emitting lasers
}

\author{
O. Buccafusca, ${ }^{\text {a) }}$ J. L. A. Chilla, J. J. Rocca, C. Wilmsen, and S. Feld \\ Center for Optoelectronic Computing Systems and Department of Electrical Engineering, \\ Colorado State University, Fort Collins, Colorado 80523 \\ R. Leibenguth \\ AT\&T Bell Laboratories, Breiningsville, Pennsylvania 18031
}

(Received 20 March 1995; accepted for publication 1 May 1995)

\begin{abstract}
We report the observation of ultrahigh frequency oscillations of up to $240 \mathrm{GHz}$ in optically gain switched vertical cavity surface emitting lasers. These oscillations are shown to be produced by multimode emission through mode competition $(10-30 \mathrm{GHz}$ ) or mode beating (above $100 \mathrm{GHz}$ ). Although these oscillations are not related to the intrinsic modulation bandwidth, some of them could be mistaken for relaxation oscillations, calling for careful interpretation of the results of this type of experiments. The highest frequencies observed for single mode relaxation oscillations were about $9 \mathrm{GHz}$ in agreement with values of modulation bandwidth reported in the literature. (C) 1995 American Institute of Physics.
\end{abstract}

The easy integrability of vertical cavity surface emitting lasers (VCSELs) into arrays makes them an attractive choice for 2D optical interconnects. For this application, high speed VCSEL operation is an important requirement. In recent years significant effort has been dedicated to determine the speed limitations of VCSELs. ${ }^{1-4}$ The direct modulation of semiconductor lasers is limited by the response of the fieldcarrier interplay which is characterized by the device relaxation oscillations. ${ }^{5}$ Although relaxation oscillation frequencies of 39 and $71 \mathrm{GHz}$ were reported in electrically gain switched VCSELs, ${ }^{3,4}$ the maximum achieved modulation bandwidth to date is of the order of $14 \mathrm{GHz}^{2}$ In this letter, we report that gain VCSELs can produce high frequency oscillations, not related to the relaxation oscillations, which can distort the measurement of the intrinsic modulation bandwidth of these devices. These oscillations are shown to be due to the presence of higher order transverse modes and were mainly observed in two frequency regions: one in the range of $10-30 \mathrm{GHz}$ due to transverse mode competition, and the other above $100 \mathrm{GHz}$ due to mode beating. We present spectrally and temporally resolved measurements of the output of optically gain switched VCSELs showing the contribution of higher order transverse modes in the high speed response of these lasers.

The devices under study ${ }^{6}$ were top surface emitting, gain guided, GaAs/AlGaAs multiple quantum well VCSELs with distributed Bragg reflectors (DBRs) consisting of 32 and 23 periods of AlAs/AlGaAs step graded layers. Measurements were performed in devices with two different active region diameters: 8 and $15 \mu \mathrm{m}$. In order to observe relaxation oscillations, the VCSELs were biased above threshold (1-1.4 times the threshold current $I_{\text {th }}$ ) and optically pumped using a mode locked Ti:sapphire laser which produces pulses of the order of $100 \mathrm{fs}$ at a repetition rate of $80 \mathrm{MHz}$. The excitation wavelength was tuned to about $780 \mathrm{~nm}$ in order to pump the quantum wells of the active area and avoiding the high re-

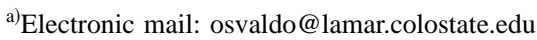

flectivity spectral region of the top DBR. The output of the VCSEL was upconverted ${ }^{7}$ in a $1 \mathrm{~mm}$ lithium lodate crystal and detected with a photomultiplier tube. The resolution, in our setup, was limited by the crystal thickness and was determined to be $160 \mathrm{fs}$ from an autocorrelation measurement of the optical pulses from the Ti:sapphire laser.

After optical excitation, the dc biased VCSELs produced an oscillatory response which fell, depending on bias and excitation parameters, into one of three regimes characterized by the frequency of the oscillation. Typical output intensity traces corresponding to each of those regimes for a 15 $\mu \mathrm{m}$ VCSEL are shown in Fig. 1. The first regime, shown in Fig. 1(a) is characterized by extremely high frequencies of more than $100 \mathrm{GHz}$. This regime was observed in several of the devices studied for high bias currents (usually greater than $1.3 I_{\text {th }}$ ) and high pulse excitation energy (greater than $0.5 \mathrm{~nJ}$ ). The amplitude of these oscillations was found to be dependent on bias, pumping energy, and temperature, but their frequency was found to be practically independent of all these parameters for a given device. Higher frequency oscillations $(>200 \mathrm{GHz})$ were observed in an $8 \mu \mathrm{m}$ VCSEL, which suggested a dependence on device geometry. In this regime, the time integrated spectra of the VCSEL output revealed multimode operation for both active area diameters, thus the high frequencies were attributed to mode beating. Under this assumption, the measured frequency of $155 \mathrm{GHz}$ for the $15 \mu \mathrm{m}$ VCSELs correspond to a wavelength separation of $3.7 \AA$ and the measured frequency of $239 \mathrm{GHz}$ for the $8 \mu \mathrm{m}$ VCSELs correspond to a wavelength separation of 5.7 $\AA$. These values are in very good agreement with the measured mode separation of $3.5 \pm 0.5$ and $6 \pm 0.5 \AA$ for the 15 and $8 \mu \mathrm{m}$ VCSELs, respectively. Still, in order to observe beat frequencies, both modes should be oscillating simultaneously, and this is confirmed by the spectrally and temporally resolved measurements discussed later.

A typical trace corresponding to the second regime is shown in Fig. 1(b). This regime is characterized by intermediate frequencies that in the devices under study ranged be- 

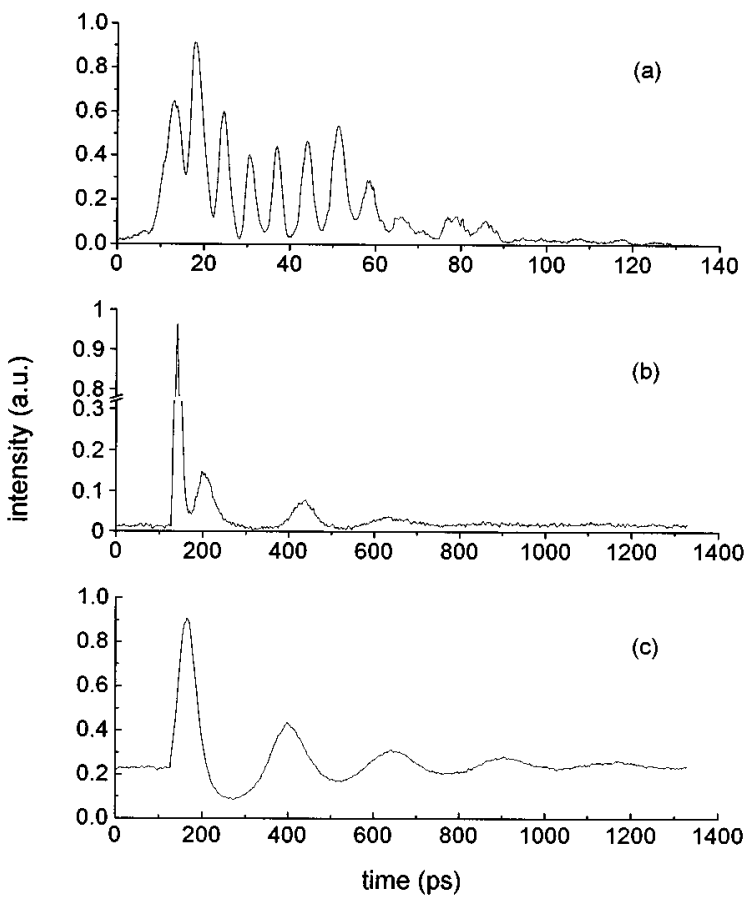

FIG. 1. Variety of oscillatory responses obtained on an optically gain switched $15 \mu \mathrm{m}$ VCSEL. (a) Ultrahigh frequency oscillations at $155 \mathrm{GHz}$ due to beating of spatial modes with 3.7 A spectral separation. (b) Intermediate frequency range $(10-30 \mathrm{GHz})$ oscillations. The gain dynamics in this case is complicated by competition of several modes for the available gain, producing a noticeably uneven separation of the peaks. (c) Lowest frequency range oscillations due to single mode relaxation oscillations. The frequency of the oscillation is constant and its amplitude shows an exponential decay.

tween 10 and $30 \mathrm{GHz}$ and was observed for bias currents greater than $1.2 I_{\text {th }}$ and pulse excitation energies greater than $0.2 \mathrm{~nJ}$. If only the first two peaks are considered, a frequency of $16 \mathrm{GHz}$ can be defined for this signal. This frequency was found to increase with an increase in bias current and pumping energy reaching a maximum value of about $30 \mathrm{GHz}$. Due to this dependence on pumping energy, these fluctuations could be misinterpreted as relaxation oscillations, however we have recorded more than one period of the oscillation and observed an uneven separation of the peaks. This kind of response is generated by competition between transverse modes and will be discussed later in more detail.

Finally an example of the third regime, which is observed at all bias currents for low excitation energies (lower than $0.2 \mathrm{~nJ}$ ), is shown in Fig. 1(c). These are monomode relaxation oscillations. The frequency and damping factor of these oscillations depends on bias and pumping energy. These oscillations are due to the carrier-field interplay in the VCSEL cavity which is related to the modulation bandwidth of semiconductor lasers. ${ }^{5}$ The maximum monomode relaxation oscillation frequencies observed in our experiments were about $9 \mathrm{GHz}$ in agreement with the measured modulation bandwidths in gain guided VCSELs. ${ }^{1,2}$

The results presented in Fig. 1 and time integrated spectra measurements suggest that multimode operation strongly affects the oscillatory response of VCSELs. To verify this hypothesis, the output from the VCSEL was simultaneously resolved in wavelength and time. This was achieved by in-
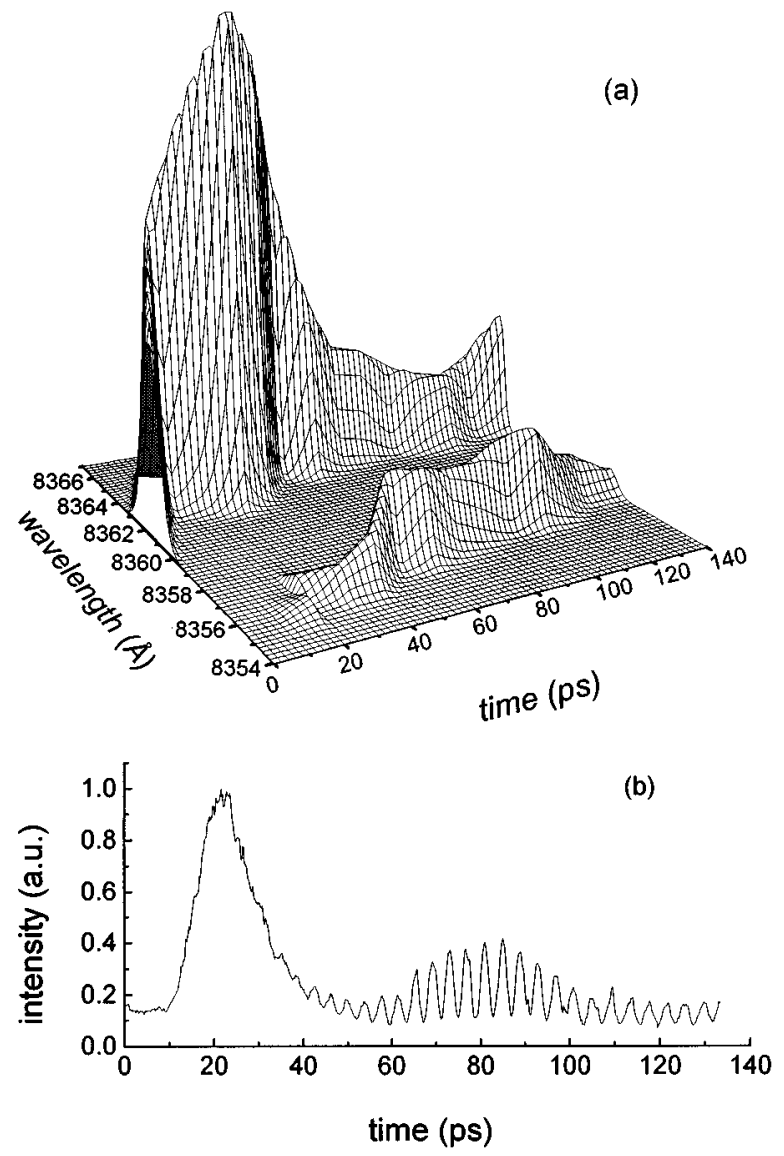

FIG. 2. (a) Time-resolved spectra measured on an $8 \mu \mathrm{m}$ VCSEL in the conditions in which ultrahigh frequency oscillations are observed. The first mode at $8361 \AA$ can be seen to start relaxation oscillations, and a second mode at $8355 \AA$ appears some time later. After 40 ps both modes have comparable amplitudes (within $10 \mathrm{~dB}$ ). (b) Total VCSEL intensity corresponding to (a) measured without spectral resolution. The ultrahigh frequency $(240 \mathrm{GHz})$ oscillations are present when the two modes in (a) have comparable amplitudes.

corporating a monochromator of half-meter focal length to select the wavelength of the VCSEL output before upconversion. However, the simultaneously spectrally and temporally resolved measurements had a joint time resolution limited by Heisenberg's uncertainty principle. For the emission wavelength of our VCSELs $(\sim 840 \mathrm{~nm})$ this limitation is given by $\Delta \lambda \Delta t=2 \AA \mathrm{ps}$, which in our case translates to a time resolution of the order of $10 \mathrm{ps}$.

The spectrum as a function of time for an $8 \mu \mathrm{m}$ VCSEL is presented in Fig. 2(a). Initially, the VCSEL is lasing at its steady-state mode which has a wavelength of $8363 \AA$ A. After optical excitation, the intensity of this mode increases and begins to oscillate at its relaxation oscillation frequency. A second mode with a wavelength of $8357 \AA$ begins to develop and, after $40 \mathrm{ps}$, reaches an amplitude comparable (within 10 $\mathrm{dB}$ ) to that of the first mode. Figure 2(b) shows the total intensity of this signal obtained by bypassing the monochromator with mirrors. The amplitude of the mode beating oscillations increases when the two modes have comparable amplitudes. The simultaneous presence of a second mode, with the wavelength separation corresponding to the ultrahigh frequencies observed and with a relative amplitude proportional to the modulation depth, confirms that the observed 


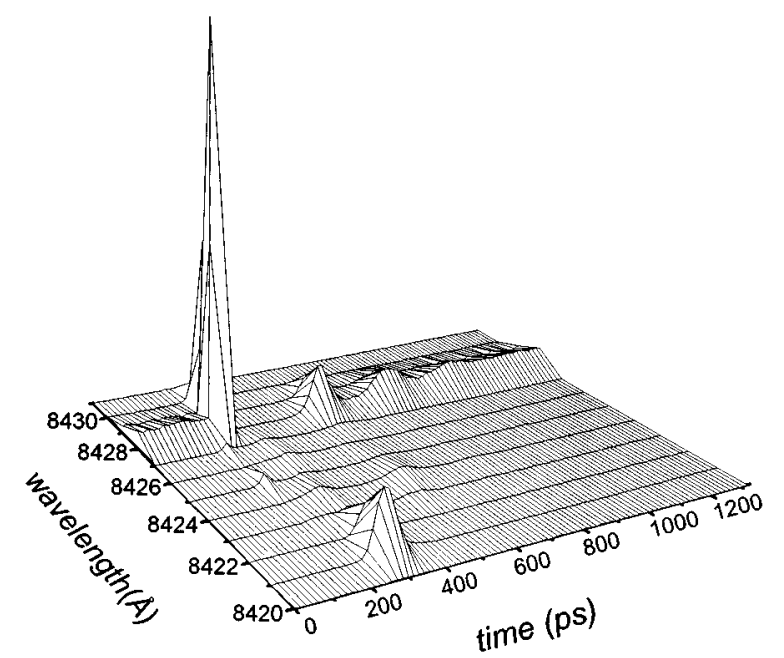

FIG. 3. Time-resolved spectra measured on a $15 \mu \mathrm{m}$ VCSEL in conditions similar to Fig. 1(b). After optical excitation the lasing mode at $8428 \AA$ displays the first peak of the relaxation oscillations, that is inhibited when a delayed pulse is emitted in the third spatial mode at $8421 \AA$. The first mode continues lasing after the higher order mode stops lasing. The contribution of the second spatial mode around $8425 \AA$ is observed to be almost negligible.

ultrahigh frequency oscillations are due to mode beating. Mode beating not only requires that the intensities of the emitting modes be comparable, but the field oscillating in both modes must also be coherent. In our experiments we found that ultrahigh frequency oscillations were produced only for a narrow range of excitation parameters (bias current between 1.3 and $1.4 I_{\text {th }}$ and energy per pulse between 0.5 and $0.7 \mathrm{~nJ}$, depending on the device). It is arguable that the necessary coherence between the modes to produce mode beating could be achieved by means of commonly used electrical excitation techniques.

While the traces shown in Figs. 1(a) and 2(b) can hardly be mistaken for relaxation oscillations, Fig. 3 suggests a mechanism through which multimode emission can mask the real relaxation oscillation frequency. Figure 3 shows the time-resolved spectrum for a situation in which only one mode dominates at each time interval. After optical pumping, the lasing mode begins to oscillate at the relaxation oscillation frequency corresponding to that mode. However when the first minimum is reached, another mode, in this case a third order transverse mode, takes over and competes for the gain in the cavity. This kind of response corresponds to a situation similar to the one depicted in Fig. 1(b). Comparing both figures it can be observed that the time difference between the first two peaks in Fig. 1(b) is not related with the interplay between the electromagnetic field in the resonator and the population inversion but with the competition of two transverse modes in the cavity. As the intensity of the pulsed excitation is increased, the delay in the onset of the second mode decreases, and accordingly the time separation between the first two peaks decreases. This can create the illusion of an increase of oscillation frequency with increasing power which can make this type of oscillation easily mistaken for monomode relaxation oscillation. A way to identify this multimode competition is by observing the usually uneven time separation between the peaks of several periods, or by observing that the amplitude envelope of the oscillations does not present an exponential decay.

In summary, we report the observation of ultrafast oscillations, with frequencies up to $240 \mathrm{GHz}$, in optically gain switched vertical cavity surface emitting lasers. These oscillations were mainly observed in two frequency regions: one in the range of $10-30 \mathrm{GHz}$ and the other above $100 \mathrm{GHz}$. Temporally and spectrally resolved measurements showed that the oscillations observed in the lower frequency range are due to transverse mode competition. Because the frequency of these oscillations increases with pumping intensity, they could, in some cases, be misinterpreted as relaxation oscillations. On the other hand, the higher frequency oscillations are generated by mode beating between two coherent transverse modes and their frequency depends only on the device geometry. As these oscillations are not produced by the carrier-field interplay in the laser cavity, they do not represent a measurement of the intrinsic modulation bandwidth of the device. The higher frequencies observed for single mode relaxation oscillations were about $9 \mathrm{GHz}$, in good agreement with the modulation bandwidths reported in the literature for gain guided VCSELs. ${ }^{1,2}$ Our results show that multimode effects can produce ultrahigh frequency oscillations in VCSELs, and can potentially obsure the determination of the relaxation oscillation frequency if spectral resolution is not utilized.

The authors wish to acknowledge Paul Armstrong for his assistance in conducting the experiments and Professor Chiao-Yao She for lending the monochromator. This work was supported by the Center for Optoelectronic Computer Systems through National Science Foundation Grant No. EEC-9015128 and by the Colorado Advanced Technology Institute Grant No. 0594.75.0738B.

${ }^{1}$ F. S. Choa, Y. H. Lee, T. L. Koch, C. A. Burrus, B. Tell, J. L. Jewell, and R. E. Leibenguth, IEEE Photonics Technol. Lett. 3, 697 (1991).

${ }^{2}$ G. Shtengel, H. Temkin, P. Brusenbach, T. Uchida, M. Kim, C. Parsons, W. E. Quinn, and S. E. Swirhun, IEEE Photonics Technol. Lett. 5, 1359 (1993).

${ }^{3}$ J. Lin, J. K. Gamelin, K. Y. Lau, S. Wang, M. Hong, and J. P. Mannaerts, Appl. Phys. Lett. 60, 15 (1992).

${ }^{4}$ D. Tauber, G. Wang, R. S. Geels, J. E. Bowers, and L. A. Coldren, Appl. Phys. Lett. 62, 325 (1993).

${ }^{5}$ A. Yariv, Optical Electronics (CBS College, New York, 1985).

${ }^{6}$ J. L. A. Chilla, B. Benware, M. E. Watson, P. Stanko, J. J. Rocca, C. Wilmsen, S. Feld, and R. Leibenguth, IEEE Photonics Technol. Lett. 7, 449 (1995).

${ }^{7}$ J. Shah, IEEE J. Quantum Electron. 24, 276 (1988). 